\title{
Medizinische Behandlungen an politischen Budgets ausrichten?
}

\author{
Jürg Schlup \\ Dr. med., Präsident der FMH
}

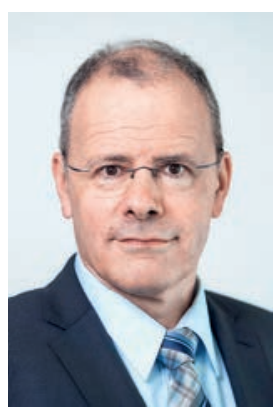

Wenn selbst der «Blick» nach einer Umfrage unter Krankenkassen nur noch ein «Prämienschöckli» erwartet [1], lässt dies auf einen milden Prämienanstieg schliessen [2]. Erwartungen weckte auch «Der Bund» mit seiner Analyse, dass die Kosten der Grundversicherung über Jahre hinweg niedriger waren als die Prämien [3]. Allein 2017 legte die Bundes-Genehmigungsbehörde Prämien fest, die um 800 Millionen Franken über den effektiven Kosten lagen [4].

Wie soll der Staat ein realistisches Globalbudget festlegen, wenn er schon bei der Prämiengenehmigung um fast eine Milliarde danebenliegt?

Es ist verständlich, dass die Prognose von Gesundheitskosten - und damit auch die Festlegung der Prämienhöhe - mit Unsicherheiten behaftet ist: Die Kostenentwicklung hängt von vielen Faktoren ab. Angesichts der heute politisch vorangetriebenen staatlich festgelegten Obergrenze für Gesundheitskosten wirft dies jedoch auch Fragen auf: Wie soll der Staat ein realistisches Globalbudget festlegen, wenn er schon bei den Prämiengenehmigungen um fast eine Milliarde neben der Realität liegt? Nichts Anderes fordern nämlich Expertenbericht und Teile der Politik mit «verbindlichen Globalzielen» oder "verbindlichen Zielvorgaben»: Der Staat soll festlegen, wie viel die Gesundheitsversorgung im nächsten Jahr kosten darf. Die Grundidee dieses planwirtschaftlichen Ansatzes ist, dass die Politik zusammen mit der Verwaltung den Bedarf an medizinischer Versorgung besser bestimmen kann als Leistungserbringer und Patienten. Weil «die Nachfrage weitgehend angebotsinduziert» sei und Leistungserbringer «ein grosses Interesse daran» hätten «über das medizinisch notwendige Mass hinaus» [5] zu behandeln, sollen Budgetzwänge der Politik korrigierend wirken. In dieser Logik wäre der Staat fähig, den Behandlungsbedarf vorauszusehen und zwischen Notwendigem und Überflüssigem zu unterscheiden. Demnach hätten wir also im Jahr 2017 für 800 Millionen zu wenig behandelt.

Aus dem Ausland wissen wir, dass Globalbudgets in der Regel zu knapp kalkuliert werden - sie sollen ja Geld sparen. Der eindrückliche Beitrag "In der Budgetfalle» auf S. 1306 behandelt darum mit Blick auf Deutschland die «zentrale Frage, die sich in einem budgetierten Gesundheitswesen stellt», nämlich wie "sich der nahezu unbegrenzte Leistungsanspruch der Versicherten" mit «der Deckelung der Kosten dieser Versorgung» verträgt [6]. Die Antwort ist so simpel wie unangenehm: Gar nicht. Obwohl Praxen in Deutschland wegen Budgetdeckelung bereits heute bis zu 20\% ihrer Leistungen gratis erbringen, will die Politik nun längere Sprechstundenzeiten erzwingen [6].

In einem budgetierten System gilt: Eine Begrenzung der Kosten zieht zwangsläufig eine Begrenzung der Leistungen nach sich und resultiert - wie in jeder Planwirtschaft - in Wartezeiten und Bevorzugung der Privatzahler. Der Versicherungsanspruch des Prämienzahlers auf die Übernahme aller notwendigen Kosten, die über die Selbstbeteiligung hinausgehen, ist in einem solchen System dahin. In der Schweiz zeigt der Bundesrat bereits heute, wie einfach sich der Versicherungsanspruch des Prämienzahlers mit Hilfe des Arzttarifs beschränken lässt: Statt den Versicherten zu erläutern, dass sie gewisse Pflichtleistungen des KVG nicht mehr erhalten werden, verfügt er über den Tarif, dass Ärzte für diese nicht mehr bezahlt werden. Wer also z.B. längere Konsultationszeiten benötigt, braucht einen Arzt, der gratis arbeitet. Das erste Kostendämpfungspaket vollendet nun die Budgetierung per Arzttarif: Zukünftig will der Bund Versorgungsleistungen beschränken, indem er die Tarifpartner zwingt, durch Steuerungsmassnahmen "Mengen- und Kostenwachstum zu korrigieren» [7].

\section{Ein begrenztes Budget und ein unbegrenzter Leistungsanspruch sind unvereinbar}

Patientenversorgung muss höheren Ansprüchen genügen als Kostenprognosen. Darum sollte die Gesetzgebung Ärztinnen und Ärzte nicht zwingen, Behandlungen an gedeckelten Budgets auszurichten. Heute führen Kalkulationsfehler «lediglich» zu Überschüssen oder Defiziten im Folgejahr. In Zukunft würde ein zu knapp kalkuliertes Globalbudget aber unmittelbar Versorgungsengpässe verursachen. 